\title{
Optimization of Quantum Cellular Automata Majority Gate Using Multiobjective Reinforcement Learning
}

\author{
Patrick Harrington ${ }^{1}$ \\ I'Department of Mathematics \& Computer Science, Northeastern State University, Tahlequah, OK, United \\ States)
}

\begin{abstract}
The Quantum Cellular Automata (QCA) majority block gate, while more robust than the simple or classic version of the gate, is still affected by lithographic manufacturing errors that affect the polarization of each individual QCA and the block circuit. Analysis and correction of these errors has been done using Bayesian, Markovian, or neural network methodologies. The problem with learning an objective to minimize errors a priori is the inherent multiobjective nature of optimization in a network of QCA composing the majority gate. Our work attempts to solve this problem by maximizing utility to achieve Pareto optimal evaluation and correction of these objectives in order to better evaluate and correct Gaussian and non-Gaussian distribution of errors. Simulation results show greater Bayesian decision-making approach has greater reduction in standard deviation of error versus Pareto-optimization of the function for Gaussian noise; in non-Gaussian noise the Pareto-optimization method performs better for a positively charged majority gate, but worse for a negatively charged majority gate.
\end{abstract}

Keywords: Reinforcement learning, Game theory, Quantum Cellular Automata, Pareto optimization, QCA gates

\section{Introduction}

Quantum Cellular Automata as possible replacements for VLSI circuits were first proposed by researchers at the University of Notre Dame [1], [2], [3], [4], [5]. These Quantum Cellular Automata are made of quantum dots, which are larger than atoms and able to hold positive or negative magnetic charge in a way more predictable than actual atoms. In a QCA gate of any configuration, data is transmitted between quantum cells by Coulomb interaction between positively and negatively charged quantum dots in each cell, transferring charge, and data, along the array or grid [1]. They have the potential to overcome CMOS circuit size limitations. However, errors in fabrication, brought on by the very small size of QCA circuits, have limited the success so far with the use of QCA as gate replacement. One such gate, the majority gate or majority voter, is often examined in QCA analysis. Tests have shown extraordinary sensitivity to alignment errors within any QCA gate, but especially that of the majority gate, which represents a significant problem to be examined. These errors can reverse the Boolean logic of the gate itself, as well as within the gate and between each QCA. The authors in [6] proposed a block majority gate, similar to the diagram below in Fig. 3. The block majority gate is less sensitive to alignment and other manufacturing errors, especially when fabricated with a grid of at least $5 \times 4$ QCA. Still, this gate has reliability problems that plague the traditional majority gate. Solving or improved analysis of this problem represents an area of important research and our work seeks to make a positive contribution to this field. We examine work related to our approach below and put forth our algorithm and its results.

\subsection{Quantum Cellular Automata}

\section{Background}

Using quantum dots, QCA encode binary information based on their magnetic charge. Quantum dots are small groups of atoms whose size is small enough that its behavior is dominated by quantum forces, but are large enough that they may be fabricated for quantum computation. The basic unit in QCA is a cell containing four quantum dots in a square. Two electrons interrupted by barriers of potential are contained within the QCA. These barriers are used to control electron movement between quantum dots. Electron polarization sets binary encoding of the cell. If the electrons are negatively charged, then the cell contains a binary 0 value; if it is positively charged, then it contains a binary 1 . Interaction between cells is done via Coulomb forces. No electrical currents are needed. Instead cells adjacent to each other are used to transmit data via polarity. The small size, low power consumption, and great throughput give the QCA its computation power. The classic or simple majority gate takes inputs from three cells, uses the majority value of these inputs to set a device cell, which is then transmitted as output (Fig. 1).

Here, we see the three inputs having values of binary 0,0 , and 1 , respectively from top to bottom. The device cell takes the polarity of the majority of the cells, a binary 0 and transmits this to the output cell on the 
right side of the gate. The QCA output cell functions by Coulomb forces and must thus go to ground, representing the lowest energy state among the charges of the three input cells, which are fixed [3]. The ANDgate is a specific case of the majority gate that can be represented by fixing the binary value of one of the input cells to 0 , which is a polarization of -1 charge.

QCA research has become more prominent due to the shrinking size of CMOS and VLSI circuits, which are now at a scale of only a few nanometers for the circuitry. However, physical limitations cause errors at this scale. In addition to expense of manufacturing, other forces besides electric current affect reliability, making the need for alternative circuits more significant. Detailed simulations of these defects are demonstrated in [7], showing that only a QCA vertical or horizontal displacement by a few nanometers is sufficient to destroy the gate logic. QCA gates are not very tolerant to errors in manufacturing that can occur with respect to defects in assembly precision and lithography. QCA cells have also been demonstrated by [6] to be highly sensitive to cell alignment. Vertical imprecision of one-half a cell width causes error in cell interaction, leading to the opposite logical effect for any gate. Current manufacturing techniques have difficulty with the precision needed: for quantum dot diameter of $5 \mathrm{~nm}$, cell size of $20 \mathrm{~nm}$, and distance between cells of $14 \mathrm{~nm}$, the margin of horizontal error is less than $10 \mathrm{~nm}$ [6], [8] or as small as $5 \mathrm{~nm} \mathrm{[7].} \mathrm{A} \mathrm{non-functional} \mathrm{majority} \mathrm{gate} \mathrm{with}$ horizontal alignment error is shown in (Fig. 2).

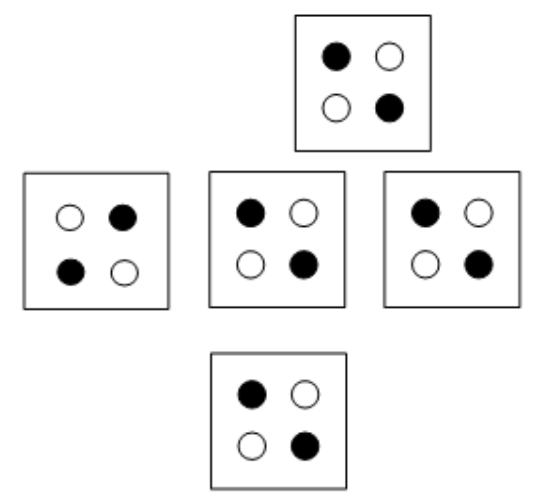

Figure 2: Non-Functional Majority Gate
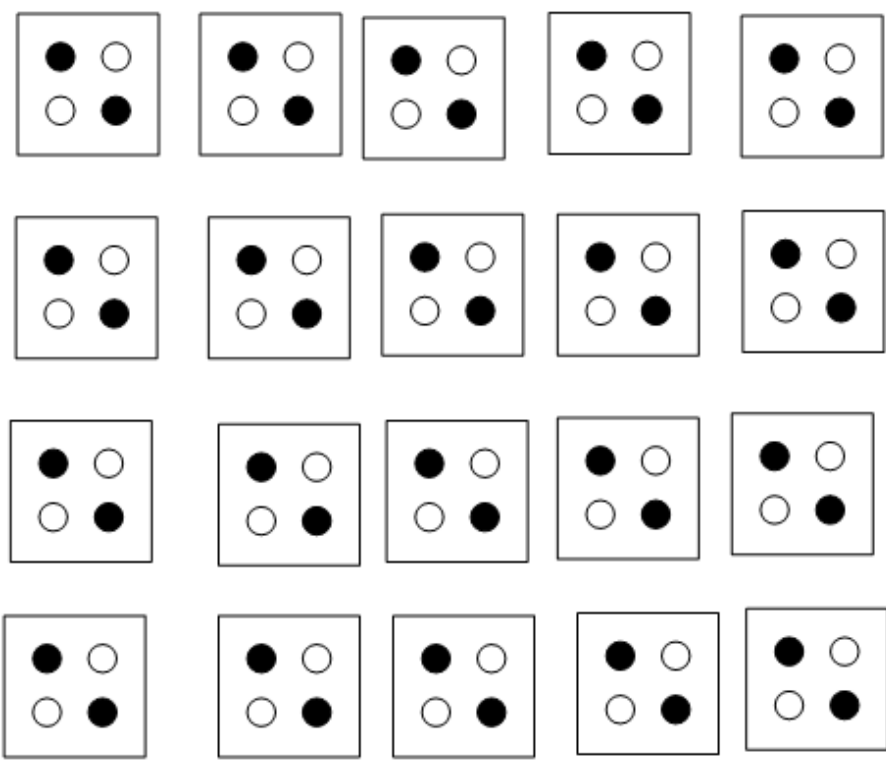

Figure 3: Block Majority Gate

The diagram (Fig. 3) shows the layout of a 5x4 block majority gate when all of the cells are laid out in a regular geometric pattern. There are three inputs which are either asymmetrical, or symmetrical as shown. The block majority gate was shown to be robust when a limited number of cells are defective, along with additional cells that are laid out irregularly. Using multiple cells (three or more) as input on each side helped keep the defective gate functional. However, changing the location and symmetry of the inputs altered the logic function of the QCA gate. There were also cases where regardless of the input, the pattern of cell misalignment and defect resulted in a non-functional block majority gate [6]. Research into addressing the problem of QCA 
gate alignment errors has been approached by [6], [7], [8], [9], [10]. Imaging algorithms have also been used in the field of manufacturing. A Markov Random Field modeling algorithm as in [11] belongs to this class of algorithms. Also, research by [9] utilized a neural network model of a QCA gate to identify device uncertainty. The authors of [12], [10] used Bayesian networks to find the probability of correct output and location of defective cells in QCA gates, but either without Gaussian error or Markov decision processes for probability calculation. Recent work at the University of Notre Dame has been done using probabilistic transfer matrices and shows the importance of the majority gate in QCA reliability [13].

\subsection{Markov Decision Processes and Bayes Classifier to minimize error}

Markov Decision Processes work to optimize reward or minimize error in environments that are partially or fully observable. For fully observable environments, an optimal policy is chosen to maximize reward or minimize cost based upon some calculated probability of reward in a stochastic environment. For current state $s_{i}$, input $a$, and next state $s^{\prime}$, it has an initial state $S_{0}$, transition equation $T\left(s, a, s^{\prime}\right)$, and reward function $R(s)$. Deciding the reward function for a transition equation appropriate to the environment given the prior data is a probability function, especially in an environment that is only partially observable and can only be described in terms of probabilistic outcomes. As such, Bayes classifiers can be used to find the most likely decision to maximize reward given the probabilistic evidence in fully or partially observable environments.

\subsection{Optimal Policy Decisions}

In an environment where each intelligent agent seeks to maximize reward in a probabilistic environment, there exists the possibility that by doing a greedy search and maximization of the environment using the more traditional processes described above, will lead to a suboptimal decision. As such, the environment description shows that each agent seeks to maximize more than one reward function. This in itself does not lend well to stochastic environments that use Gaussian error calculation variants and related probabilistic decision theory. As such, there exists the possibility that an outcome chosen by agent is suboptimal. An outcome that is Pareto optimal, however, is maximized for all players. This adds an additional probabilistic vector of decisions that must be made. In [14] the authors use a modified Pareto rule to allow for agents to collaborate to increase the possibility of maximizing their individual reward in a probabilistic environment. In fact, groups of three or more intelligent agents are much more likely to maximize group reward when agents are free to leave or join the group. It is possible for agents to play games of mixed strategies where moves are calculated as a probability of action over pure strategies.

\section{Proposed Approach}

We examine the use of evaluation and correction of QCA gate with Gaussian error over a distribution of values representing the majority gate. Our proposal will show the mathematical model and approach of applying a game theoretic solution for pure strategies for a partially observable Markov Decision Process in a probabilistic environment that has multiple objectives, in order to maximize reward, to evaluate QCA state and errors and thereby detect and correct errors by correctly evaluating the gate polarity for Gaussian and/or nonGaussian noise. Each QCA in our model acts as an intelligent agent seeking to maximize reward and minimize likelihood of error, and learns iteratively via reinforcement learning to maximize reward given prior action. Error is determined iteratively until convergence via a Bayes classification method, with game-theoretic decision making to maximize group reward for QCA organized in groups of four. Using this method to determine correct polarity value enables us to find the most likely ground state. Our method serves as an analysis tool for determining actual output of a majority gate made of Quantum Cellular Automata, with the possible existence of fabrication errors that cause polarity of each QCA to deviate from a measurable full positive or negative charge. This deviation is affected by both individual QCA alignment and polarization due to manufacturing error as well as that of its neighbors. Further complicating evaluation of the gate is due to the physics of electromagnetic charge; charge is never measured discretely negative or positive, but instead it is a continuous value over $(-1,+1)$. We adapt this to the Bayes classification method so that measured negative charge is over the range from $(0,<0.5)$ with positive charge from $(0.5,1.0)$.

Since it is unknown whether a QCA is affected by errors, we believe that the use of a game-theoretic adaptive learning algorithm that seeks to maximize reward in an environment that works with unknown existence of Gaussian error and/or non-Gaussian error, and unknown action by the other intelligent agents, could be effective. We apply this to the QCA majority gate and represent error as a Gaussian distribution over the block gate. Non-Gaussian error, as shown below, is represented by reverse polarity of a 3x3 block of QCA. The objective of our algorithm is not just maximization or minimization of the value of the charge to find the correct signal or polarity, but it works to account for fabrication errors or possible actual value of charge for one or more QCA being different from that of its neighbors. Our algorithm removes noise from a signal for finding correct value of a QCA using Pareto-optimal game theoretic action for pure strategies to maximize reward given 
prior probable QCA charge. In our work we only consider the group to be neighbors to the immediate left, right, top, and bottom. To perform error analysis of the block QCA majority gate, each QCA and its charge relative to its neighbors will be examined. Once error is removed using a game-theoretic process, analysis of standard deviation and average majority gate value is given. From here, threshold values could be easily applied to the gate itself; that is, since the entire system of cells makes up the logic gate, if the entire system of QCA is above the threshold value of 0.5 for a given gate, then the corresponding positive charge (Boolean value of 1 ) is output, with a negative charge (Boolean value 0) output otherwise. For given node the QCA that make up the majority gate of size $m \times n$ is:

$X=\sum x_{i, j}$

$X$ is modeled as a vector of joint distribution of probability values with Gaussian noise. For each QCA, with a given QCA denoted as $x_{i j}$ has four neighbors:

$\left\{x_{k, l}\right\} \in X,\left\{x_{k, l}\right\} \subseteq X$

Probability of error is given using a posterior probability distribution over $X$. Thus each $\left\{x_{k, l}\right\}$ is used to calculate likelihood of certainty each QCA polarization value being correct:

$\left\{x_{k, l}^{\prime}\right\} \in X^{\prime}$

using a priori method and Bayes classification:

$\frac{P\left(x_{i, j} \mid x_{i, j}^{\prime}\right) P\left(x_{i, j}^{\prime}\right)}{P\left(x_{i, j}\right)}$

Distribution of error is given by:

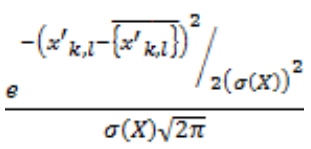

Utility of $\left\{x_{k l}^{\prime}\right\}$ for game theoretic analysis for pure strategies is given by

$$
\operatorname{Argmax}\left(\frac{P\left(x_{k l} \mid x_{k l}^{l}\right) P\left(x_{k l}^{l}\right)}{P\left(x_{k l}\right)}-\frac{e^{-\left(x_{k l l}^{\prime}-\overline{\left(x_{k l l}^{\prime}\right)}\right)^{2}} / 2(\sigma(X))^{2}}{\sigma(X) \sqrt{2 \pi}}\right)
$$

\section{Results}

We model the QCA gate as a 5x5 grid of simulated QCA having values with Gaussian noise added to each representative cell. Groups range from $0 . . .4$ depending on optimal policy per agent. Evaluating 500 circuit models, each having unique Gaussian noise distribution, we examined average and standard deviation of each. While the average was, as expected, not a useful tool in error analysis, standard deviation was. We compare our Pareto-optimal game-theoretic algorithm for pure strategies with the same algorithm that does not play a game of Pareto-optimality. In (Fig. 4 - 7) the QCA is assumed in our test cases below to have a Boolean value of 1. After noise is removed, standard deviation shrinks by an order of magnitude but there is no measureable change in polarization, as the average polarization with noise was Boolean 1, and it was still Boolean 1 after noise removal. 


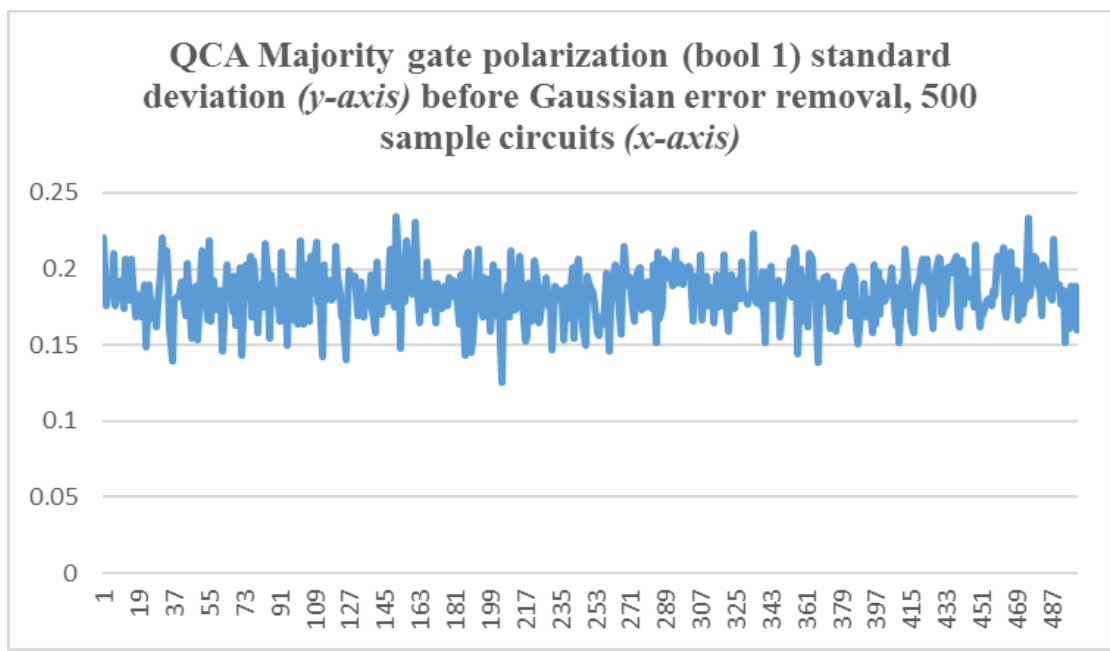

Figure 4: Standard Deviation, Boolean 1 Majority Gate Polarization Standard Deviation Over 500 Sample Circuits With Gaussian Error

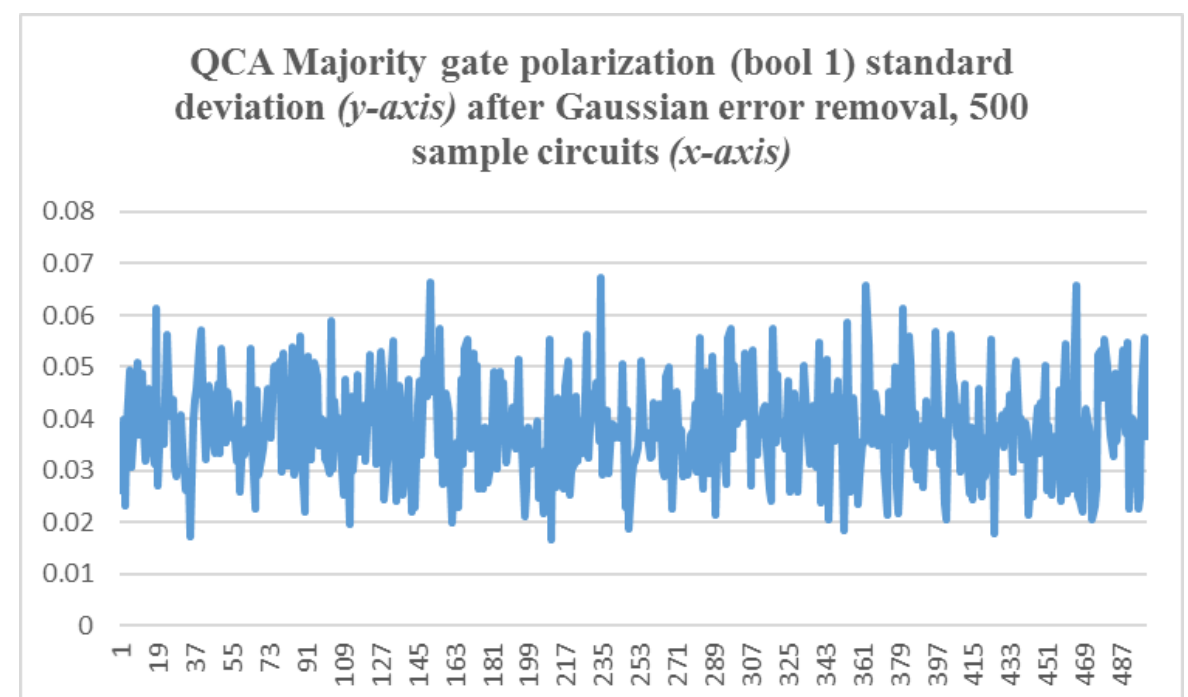

Figure 5: Standard Deviation, Boolean 1 Majority Gate Polarization Over 500 Sample Circuits After Error Correction

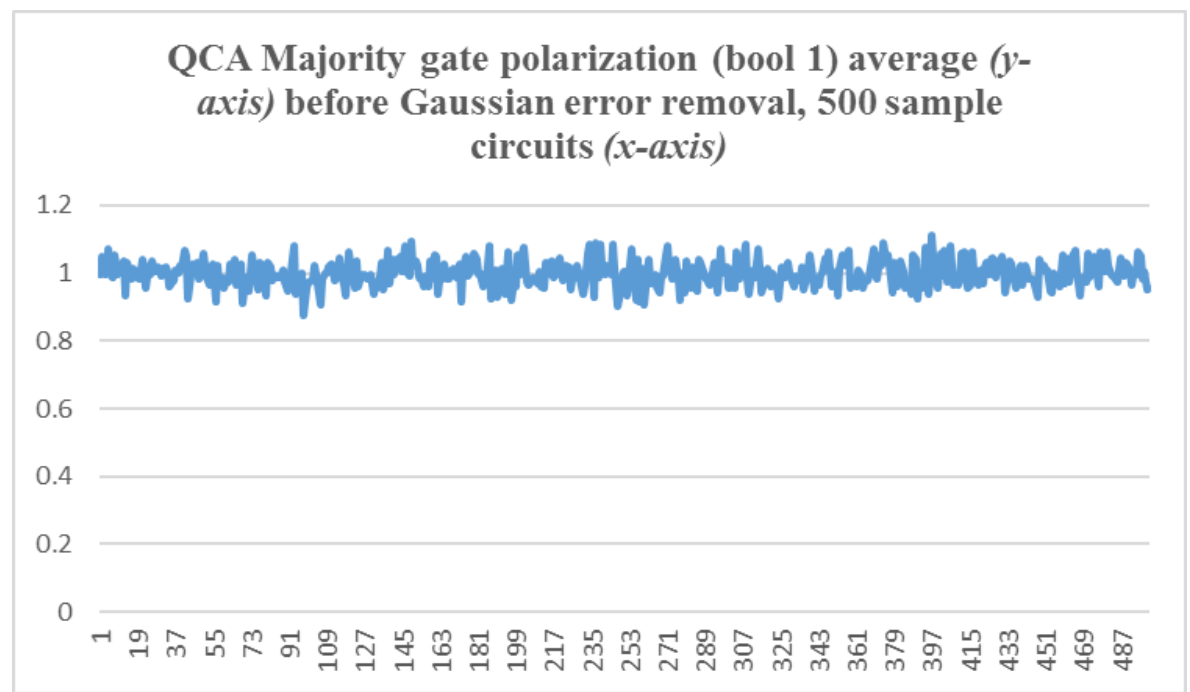

Figure 6: Average Polarization, Boolean 1 Majority Gate Over 500 Sample Circuits With Gaussian Error 


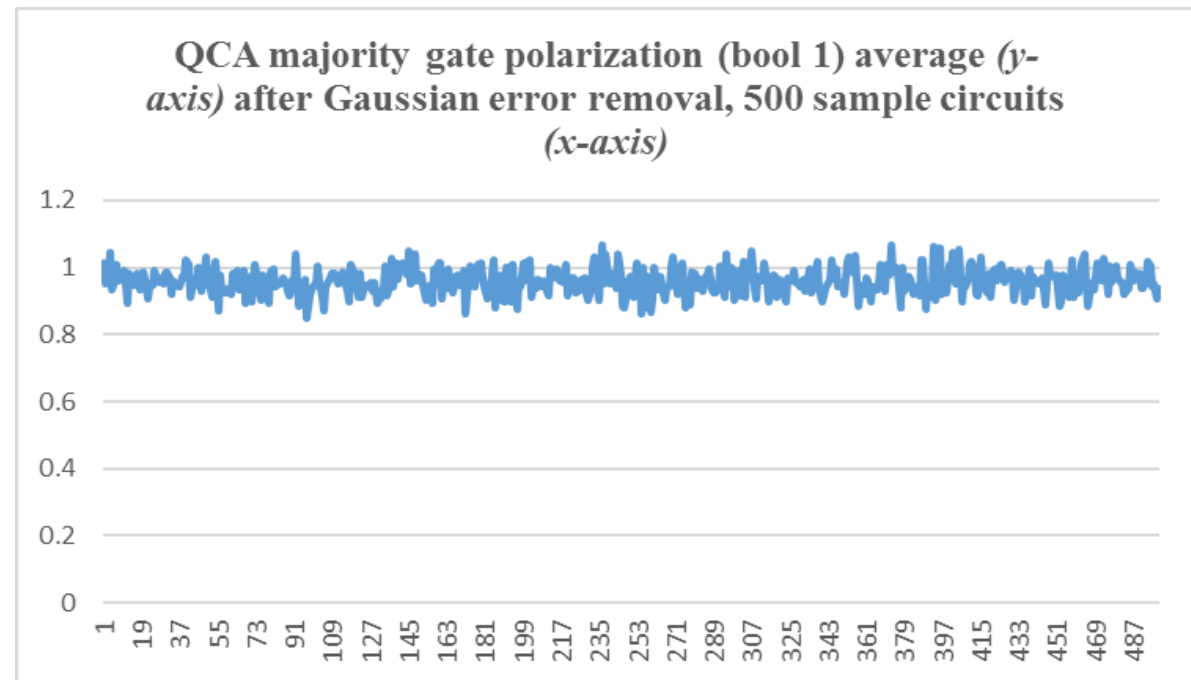

Figure 7: Average Polarization, Boolean 1 Majority Gate Over 500 Sample Circuits After Error Correction

Below in (Fig. 8 - 11) we see the same algorithm for each QCA set to Boolean zero, again as above each QCA having Gaussian noise representing measurable polarization error. Once noise is removed, we see similar results as above: standard deviation gets smaller nearly by an order of magnitude, and polarization behaves the same as it did with QCA Boolean 1:

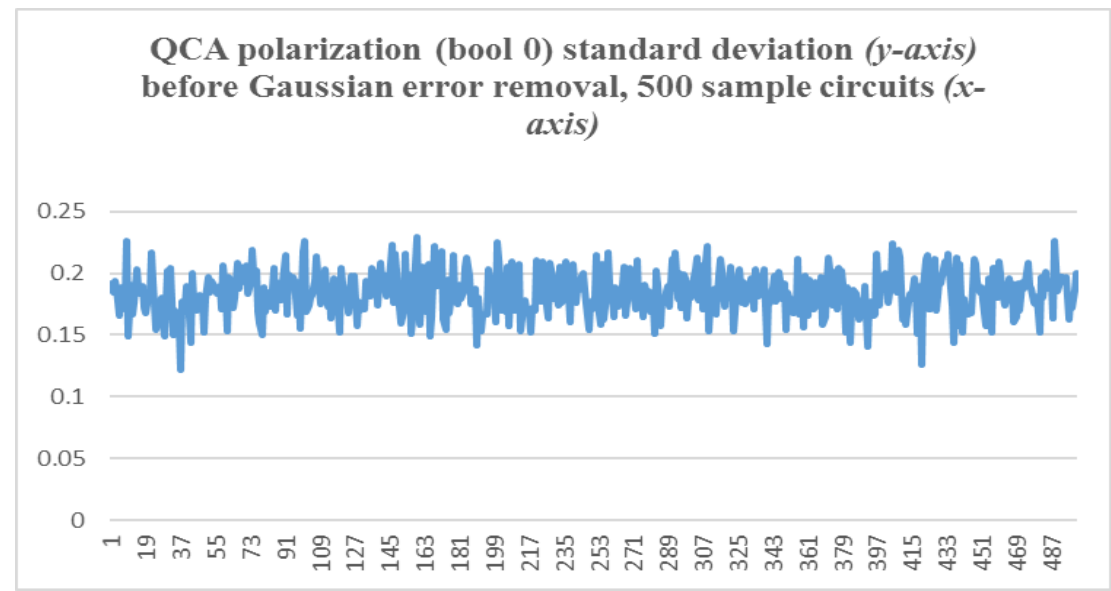

Figure 8: Standard Deviation, Boolean 0 Polarization Of Majority Gate Over 500 Sample Circuits Before Error Correction

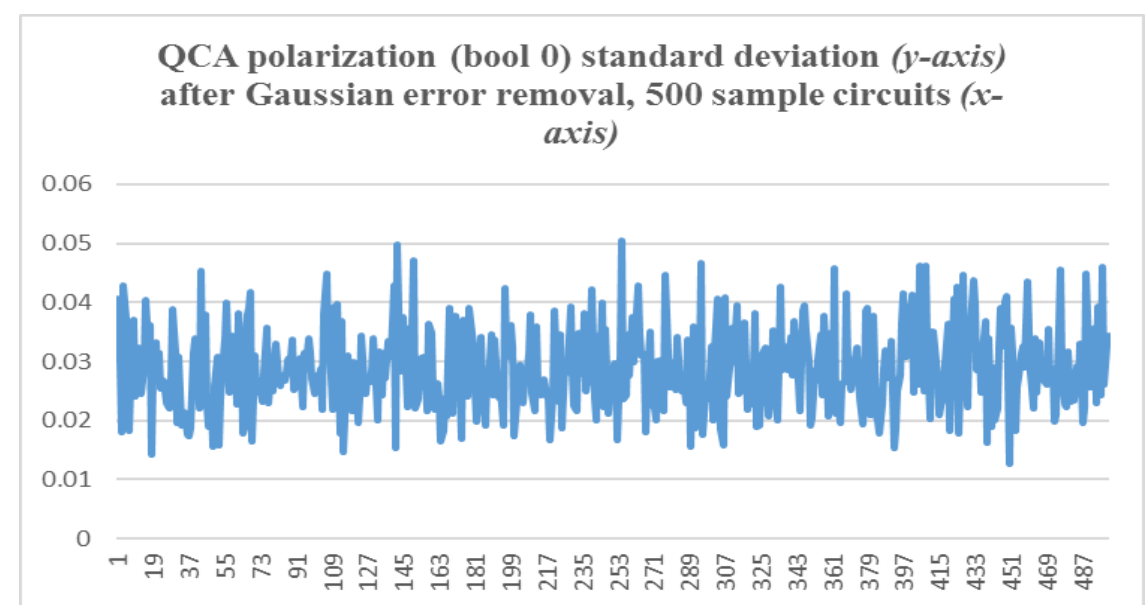

Figure 9: Standard Deviation, Boolean 0 Polarization Of Majority Gate Over 500 Sample Circuits After Error Correction 


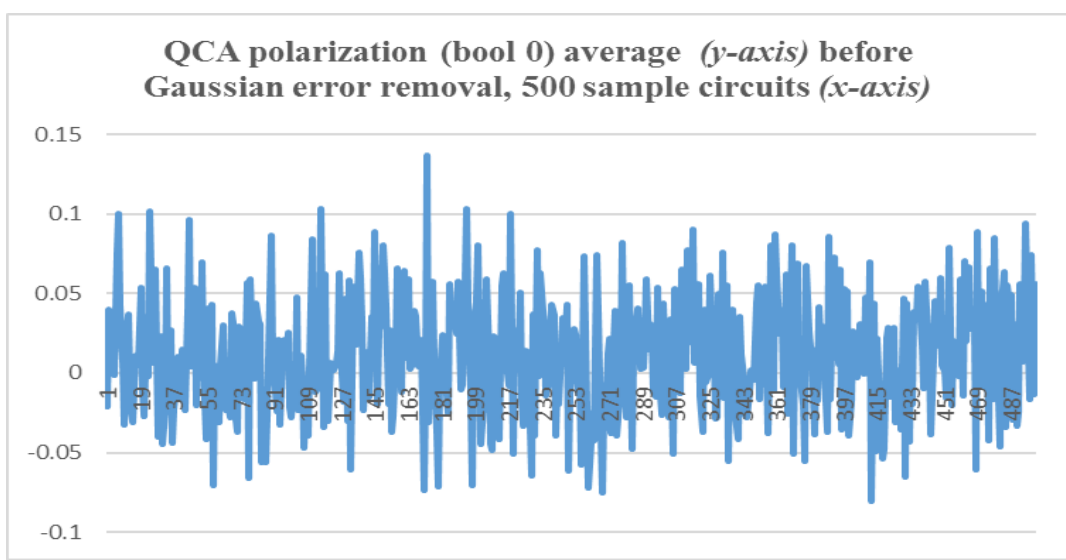

Figure 10: Average Polarization, Boolean 0 Majority Gate Over 500 Sample Circuits Before Error Correction

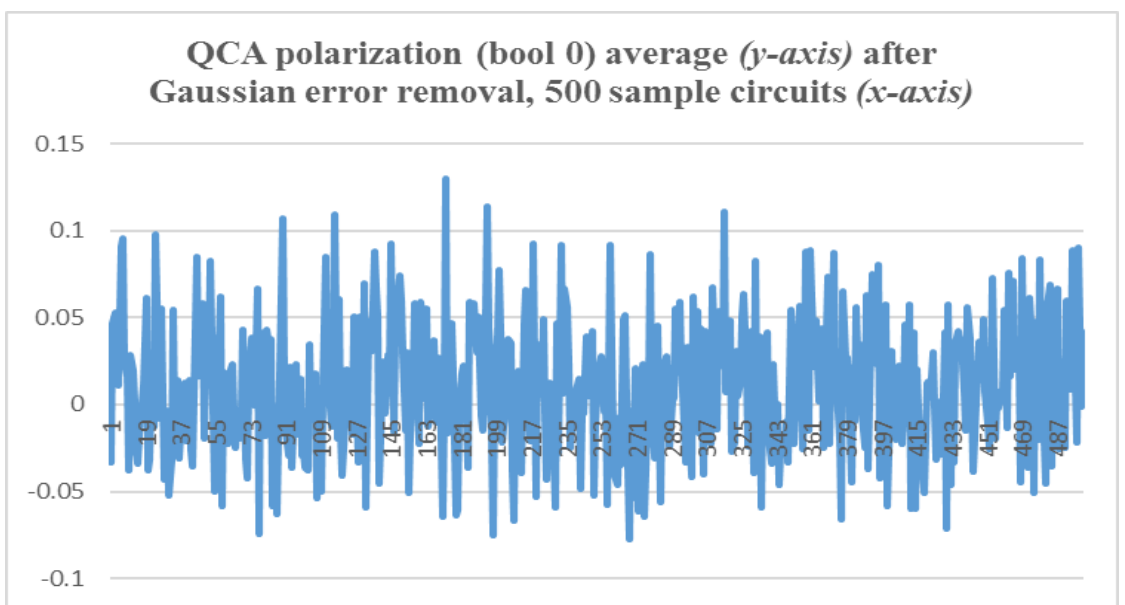

Figure 11: Average Polarization, Boolean 0 Majority Gate Over 500 Sample Circuits After Error Correction

Next in (Fig. 12 - 13) we examine Pareto-optimized error correction. For Boolean 1 and Boolean 0, standard deviation was similar between the two after Pareto-optimization. However, our non-Pareto optimized approach did better at reducing standard deviation. In fact, standard deviation was significantly reduced between QCA after application of our non-Pareto optimized algorithm. Standard deviation of the QCA gates with Gaussian error was approximately 0.2 over the 500 simulated test cases. However, once our algorithm without Pareto-optimization was applied standard deviation shrunk by nearly one-tenth to approximately 0.04 over the same 500 simulated test cases. With Pareto-optimization it was twice as large at 0.08 . This is interesting as we expected the standard deviation after Pareto-optimization to be smaller. Average polarization (Fig. 14 -15) was similar to above and stayed at the relative expected Boolean values:

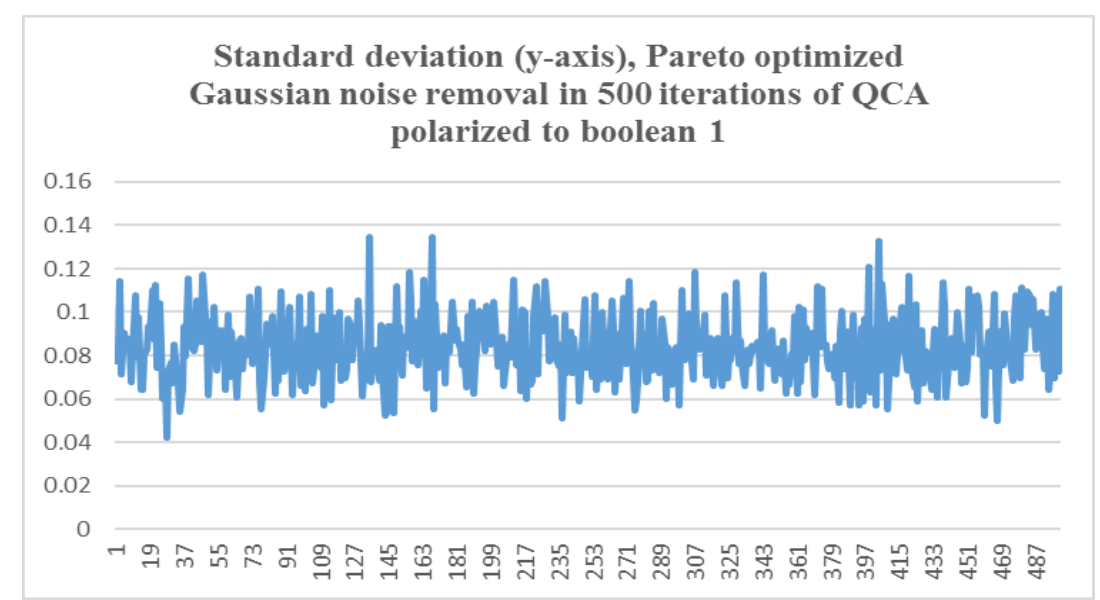

Figure 12: Standard Deviation, Boolean 1 Polarization Of Majority Gate Over 500 Sample Circuits After Pareto-Optimized Error Correction 


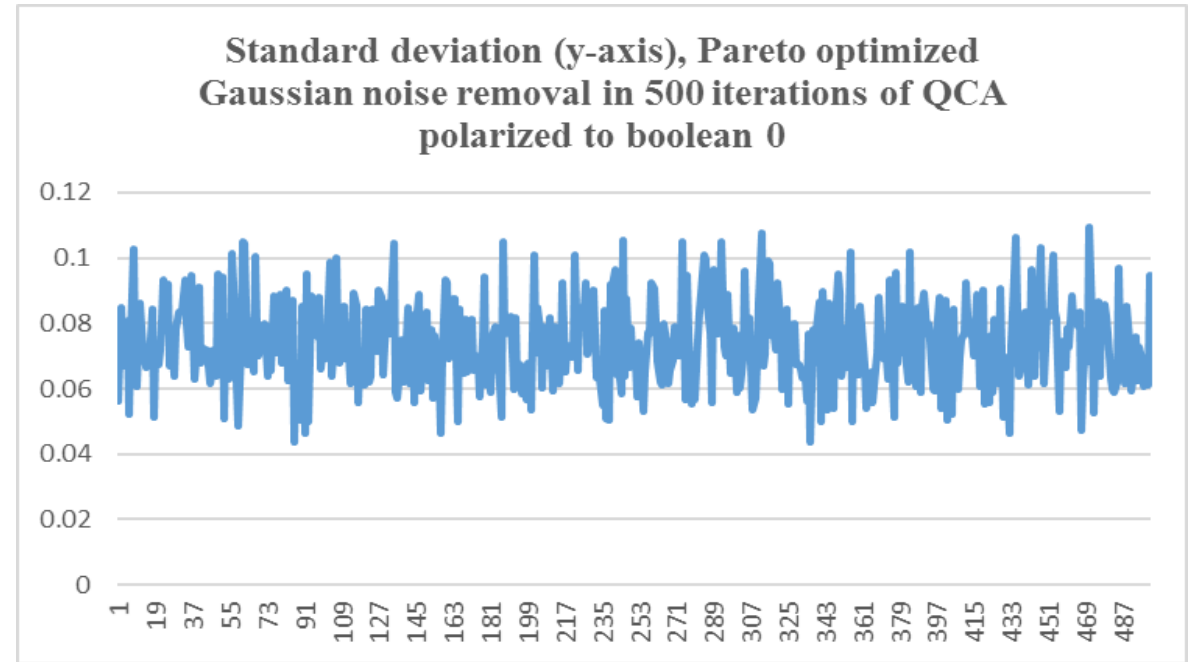

Figure 13: Standard Deviation, Boolean 0 Polarization Of Majority Gate Over 500 Sample Circuits After Pareto-Optimized Error Correction

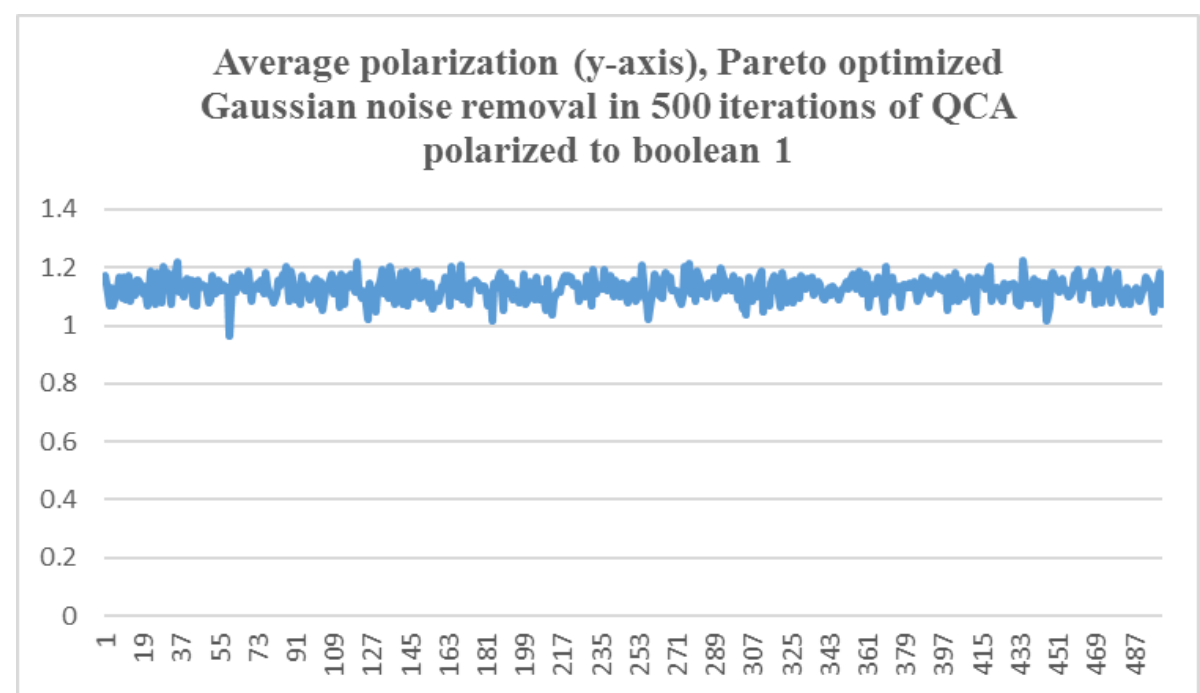

Figure 14: Average Polarization, Boolean 1 Polarization Of Majority Gate Over 500 Sample Circuits After Pareto-Optimized Error Correction

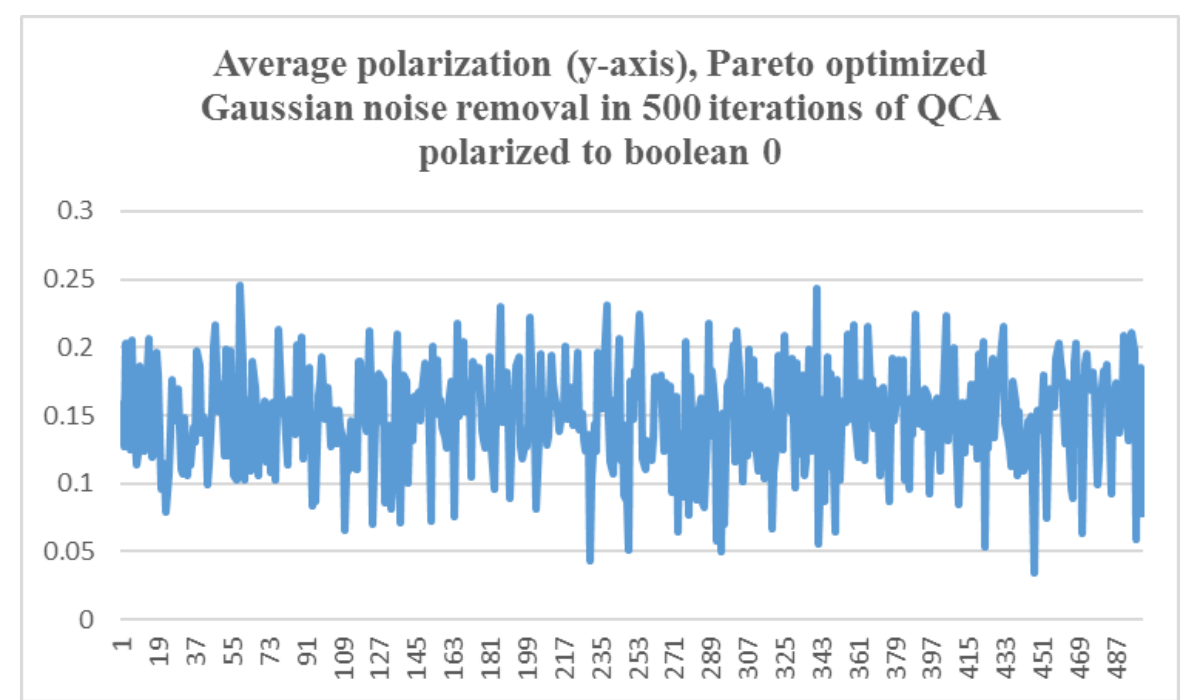

Figure 15: Average Polarization, Boolean 0 Polarization Of Majority Gate Over 500 Sample Circuits After Pareto-Optimized Error Correction 
In the following diagrams we examine how our Pareto and non-Pareto algorithms work with nonGaussian noise. The results below show the addition of non-Gaussian noise, along with the Gaussian noise as in the previous results. Non-Gaussian noise is represented as a failure of reverse polarity for a 3x3 QCA block of cells. (Fig. 16, 17) show the standard deviation and average, respectively, of a gate with Gaussian and nonGaussian noise:

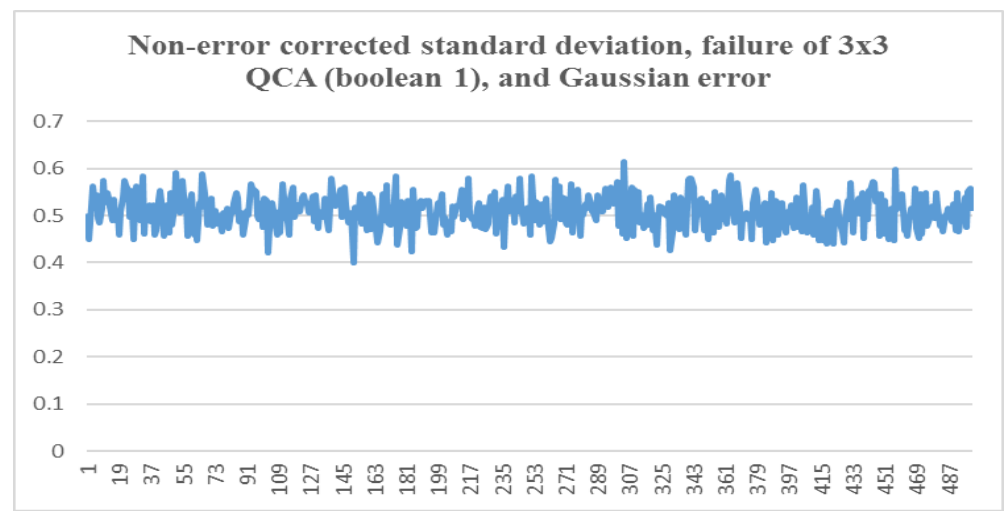

Figure 16: Standard Deviation, Non-Gaussian And Gaussian Noise, Semi-Functional QCA In Gate Set To Boolean 1 Polarization Over 500 Sample Circuits Before Error Correction

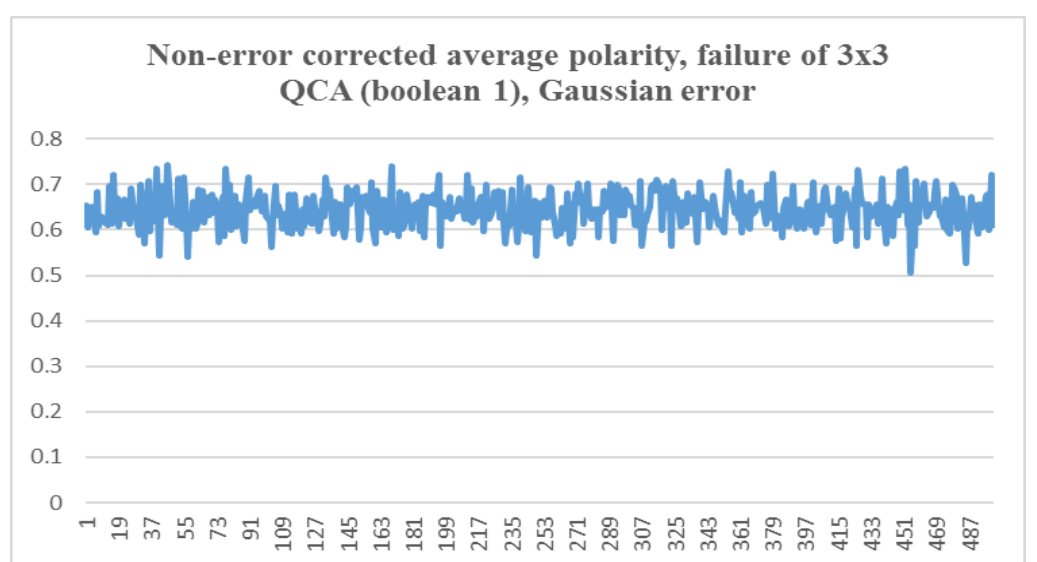

Figure 17: Average Gate Polarity, Non-Gaussian And Gaussian Noise, Semi-Functional QCA In Gate Set To Boolean 1 Polarization Over 500 Sample Circuits Before Error Correction

In (Fig. 18, 19) we see our non-decision-theoretic algorithm's performance with the non-Gaussian and Gaussian noise. (Fig. 18) shows standard deviation significantly reduced. (Fig. 19) shows greater magnitude of variance with respect to polarity versus before error removal. Overall, the average polarity variance went closer to Boolean 1.

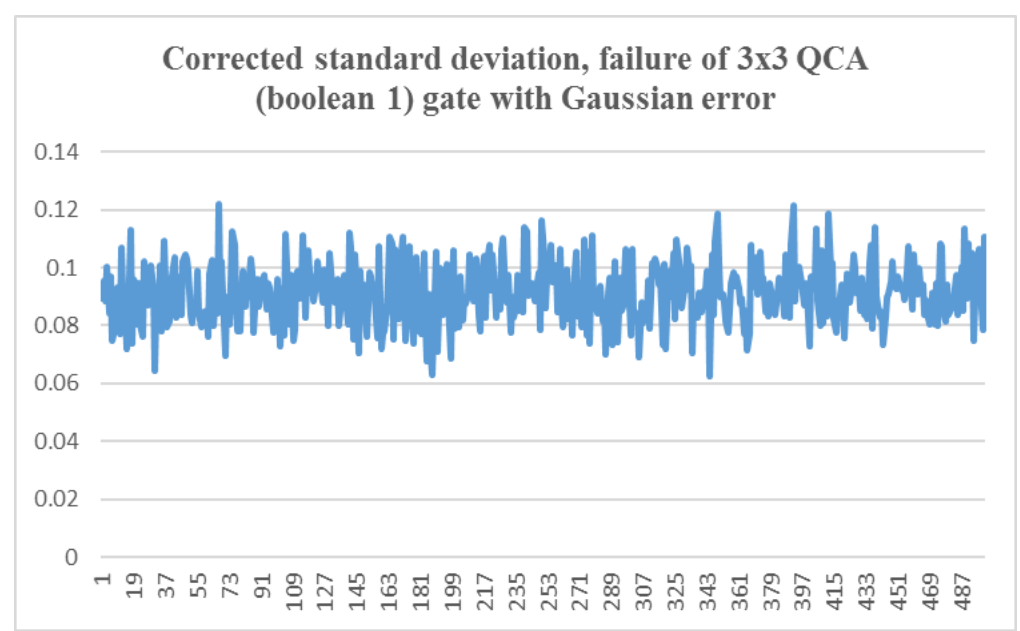

Figure 18: Standard Deviation, Non-Gaussian And Gaussian Noise, After Error Correction, Semi-Functional QCA In Gate Set To Boolean 1 Polarization Over 500 Sample Circuits. 


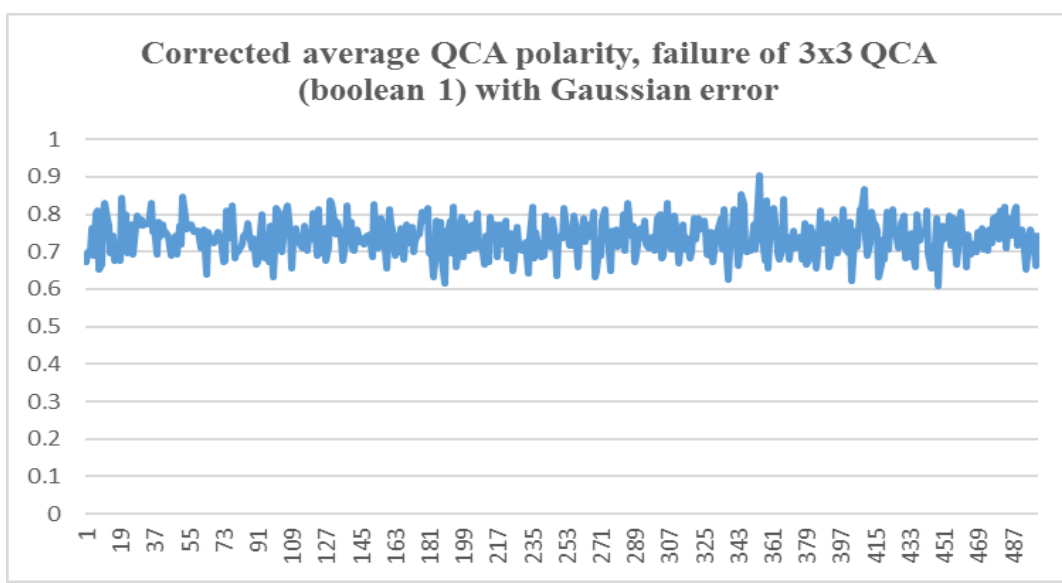

Figure 19: Average Gate Polarity After Error Correction Of Non-Gaussian And Gaussian Noise, SemiFunctional QCA In Gate Set To Boolean 1 Polarization Over 500 Sample Circuits.

In (Fig. 20, 21) below we see how our Pareto-optimized algorithm removes Gaussian and nonGaussian noise. Note that standard deviation (Fig. 20) was greater for our Pareto algorithm than our non-Pareto algorithm in (Fig. 18). However, our Pareto-optimized algorithm had a higher polarity (Fig. 21) than our nonPareto algorithm in (Fig. 19):

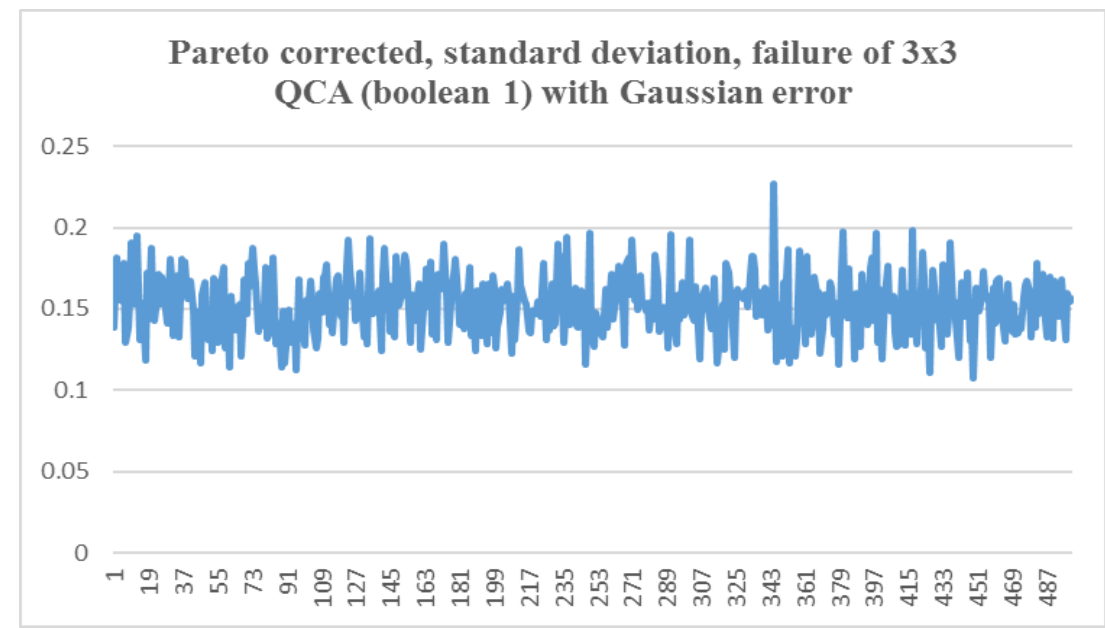

Figure 20: Standard Deviation After Error-Correction Of Non-Gaussian And Gaussian Noise For SemiFunctional QCA In Gate Set To Boolean 1 Polarization Over 500 Sample Circuits

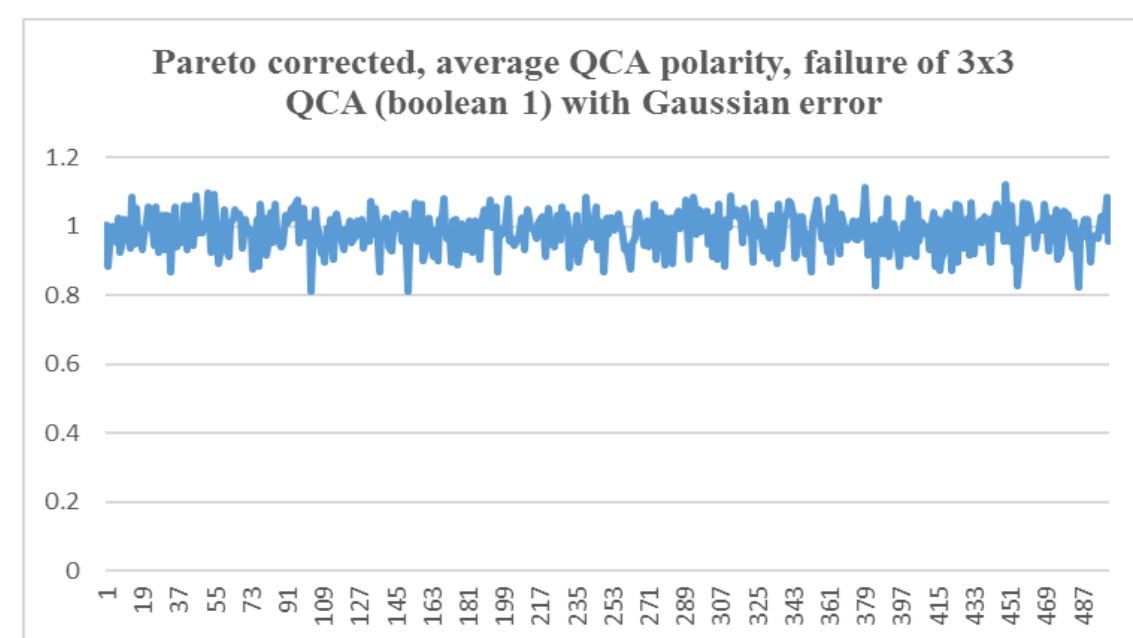

Figure 21: Average Polarity Of Gate After Pareto-Optimized Gaussian And Non-Gaussian Noise Removal, Functional QCA In Gate Set To Boolean 1 Polarization Over 500 Sample Circuits Before Error Correction. Note The Average Gate Polarity Has Been Restored To The Correct Boolean Value Of 1. 
In (Fig. 22 - 27) below we see the same comparison but for QCA gates set to Boolean 0. Again we have non-Gaussian noise (3x3 QCA block failure represented by reverse polarity) added to the Gaussian noise (Fig. 22). In (Fig. 23) we see standard deviation of our non-Gaussian algorithm after noise is removed. As with Boolean 1 above, the non-Pareto algorithm (Fig. 23) had nearly twice as small standard deviation than our Pareto-optimized algorithm (Fig. 24). Note that while our Pareto optimized algorithm performed similarly as it did with Gaussian noise, our non-Pareto optimized algorithm performed worse with the addition of nonGaussian noise:

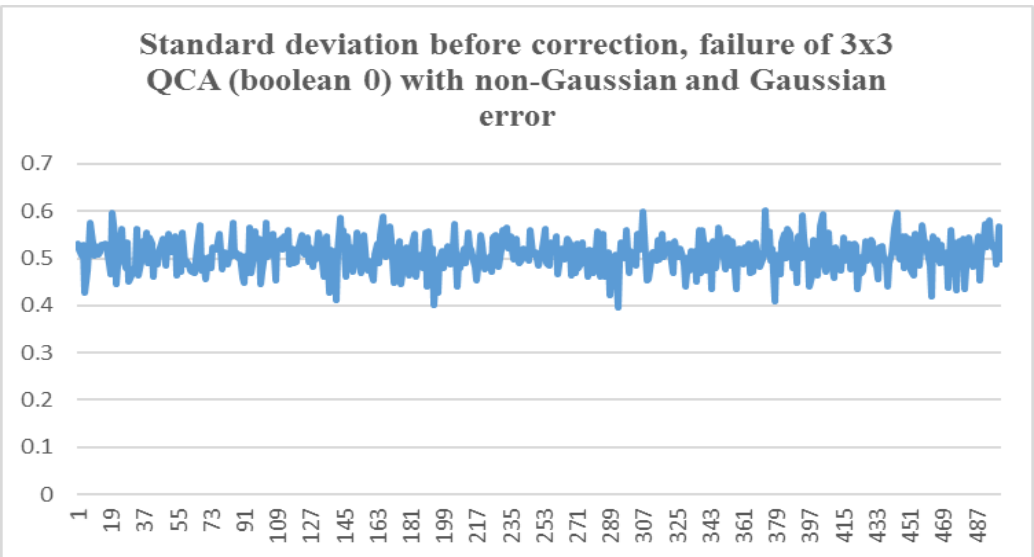

Figure 22: Standard Deviation Of Gate Before Non-Gaussian And Gaussian Error Correction, Semi-Functional QCA In Gate Set To Boolean 0 Polarization Over 500 Sample Circuits.

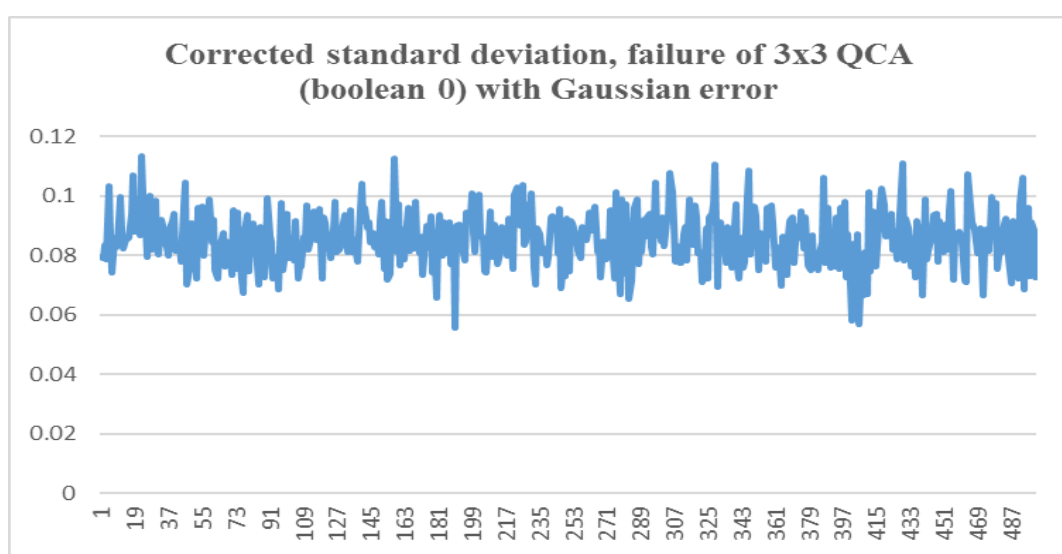

Figure 23: Standard Deviation Of Gate After Non-Gaussian And Gaussian Error Correction, Semi-Functional QCA In Gate Set To Boolean 0 Polarization Over 500 Sample Circuits.

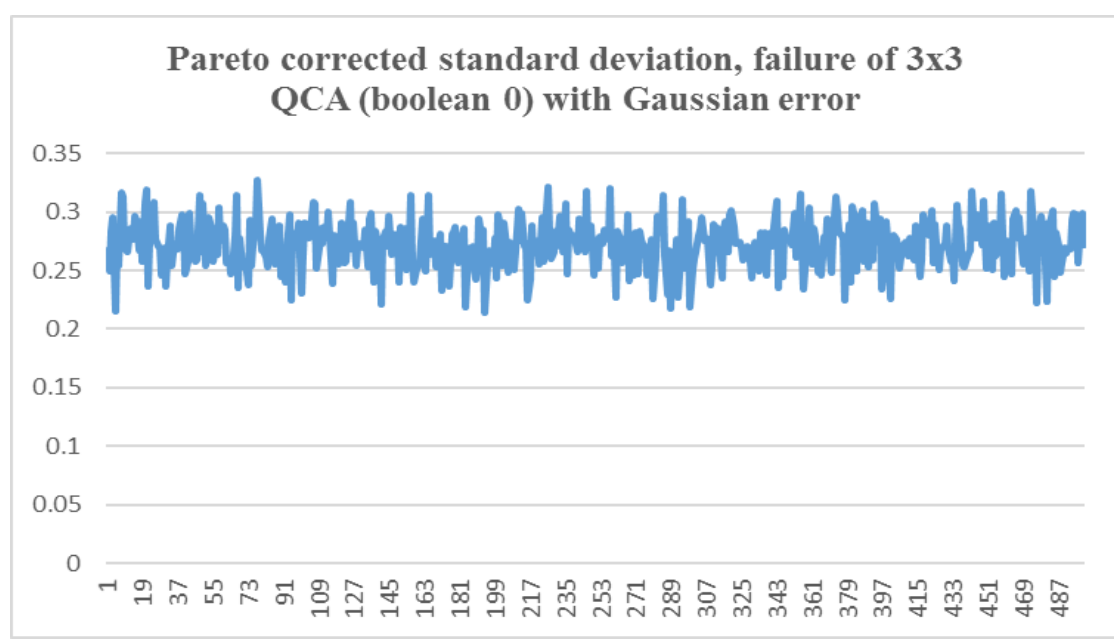

Figure 24: Standard Deviation Of Gate Polarity After Pareto-Optimized Non-Gaussian And Gaussian Error Correction, Semi-Functional QCA In Gate Set To Boolean 0 Polarization Over 500 Sample Circuits. 
Finally, we see the average majority gate polarity after non-Gaussian and Gaussian noise is removed by our non-Pareto and Pareto-optimization algorithms. Average polarity of our non-Pareto algorithm (Fig. 26) moved closer to polarity of binary 0 compared to gate polarity prior to noise removal (Fig. 25). However, the polarity of the gate went towards binary 1, which is the wrong direction, after our Pareto-optimized algorithm attempted to remove Gaussian and non-Gaussian noise (Fig. 27):

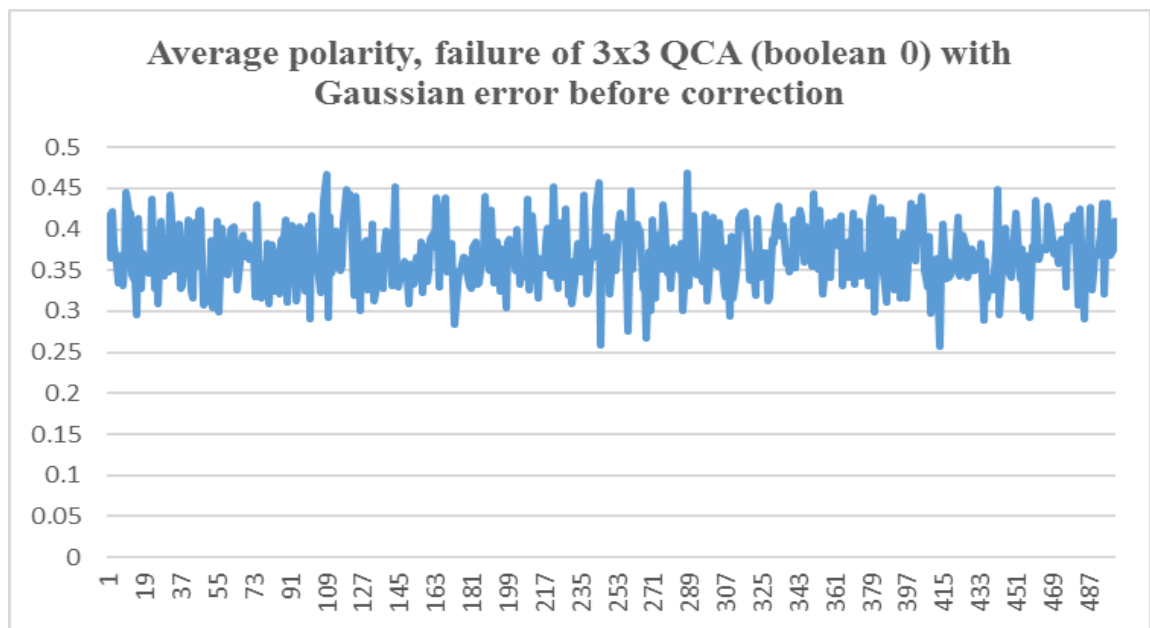

Figure 25: Average Gate Polarity Before Non-Gaussian And Gaussian Error Correction, Semi-Functional QCA In Gate Set To Boolean 0 Polarization Over 500 Sample Circuits.

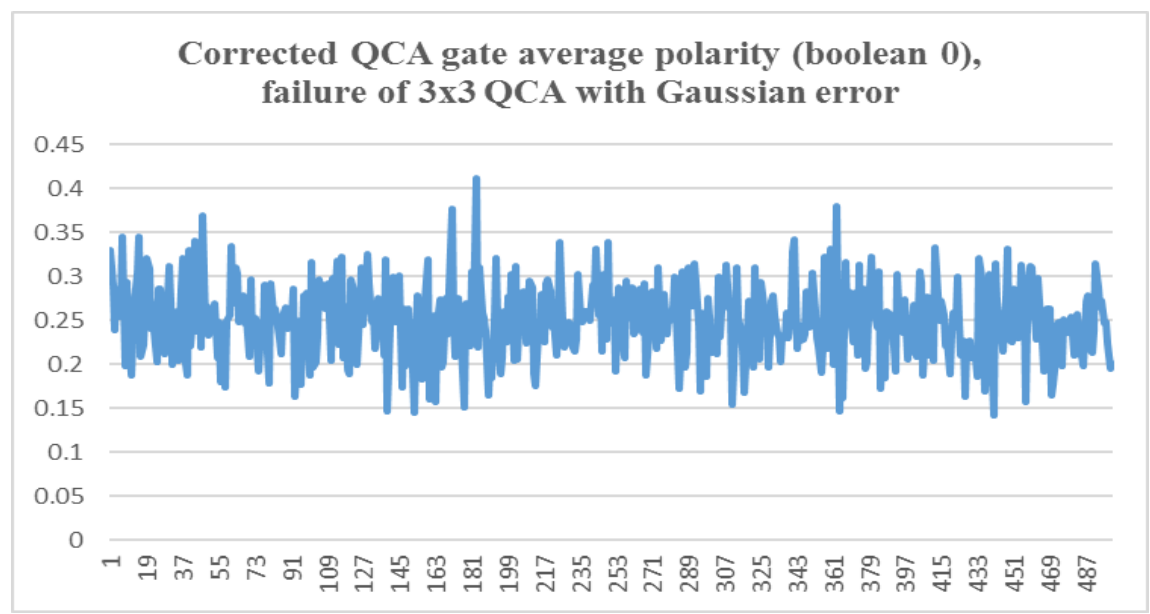

Figure 26: Average Polarity Of Gate After Non-Gaussian And Gaussian Error Correction, Semi-Functional QCA In Gate Set To Boolean 0 Polarization Over 500 Sample Circuits.

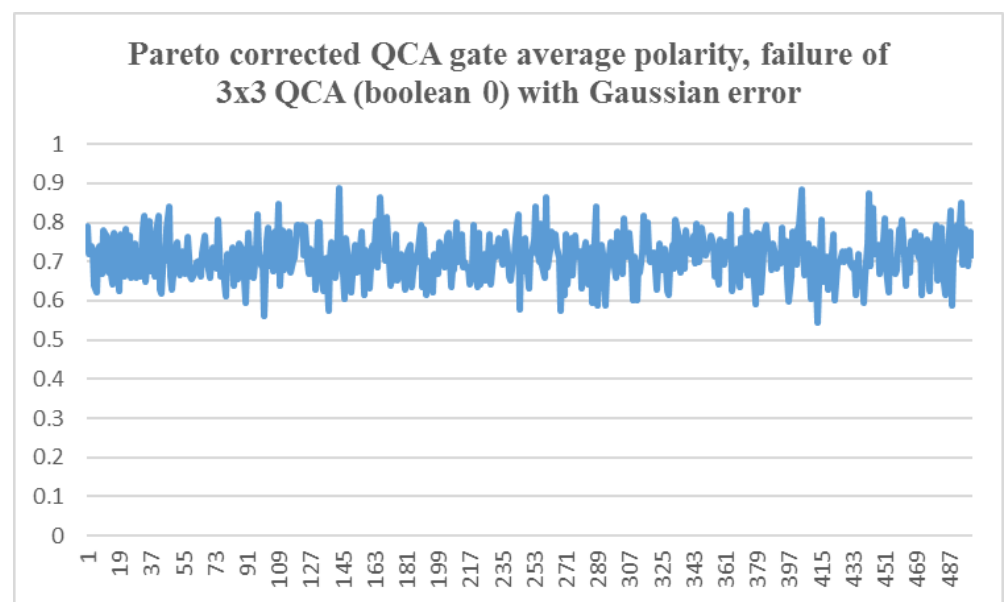

Figure 27: Average Polarity Of Gate After Pareto-Optimized Non-Gaussian And Gaussian Error Correction, Semi-Functional QCA In Gate Set To Boolean 0 Polarization Over 500 Sample Circuits. 
We were surprised to find that our Pareto-optimized algorithm performed worse for the Boolean 0 QCA gate, but better for the Boolean 1 QCA gate. Although in terms of standard deviation reduction, our nongame-theoretic approach of Gaussian error minimization performed better when faced with pure Gaussian error, the game-theoretic approach of Pareto-optimization outperformed our non-game-theoretic approach significantly when faced with non-Gaussian error represented by QCA failure in a 3x3 block in addition to Gaussian error for Boolean 1, and showed a greater consistent standard deviation. In this, the Pareto-optimized method was able to restore the average polarity of the gate to Boolean 1. The Gaussian error minimization did not significantly change the average polarity. However, when faced with Boolean 0, the Pareto-optimal solution seems to have maximized the expected polarity of the gate to a Boolean value of 1 .

\section{Conclusion}

Our solution builds upon previous work using Bayes classification, Markov modeling, and Pareto optimization to identify and correct fabrication and resultant polarization errors in a QCA majority gate. Errors are represented as Gaussian noise over a distribution of values, as well as that of a $3 \times 3$ grid of QCA failure for reverse polarity. We believe we should continue to examine cases for mixed strategy game-theoretic Pareto optimization, and extend the work beyond the pure strategies shown above. While our work is purely a beginning, we believe it is indicative of the need for further exploration of Pareto-optimization and gametheoretic optimization of multi-objective problems. Our work shows the possibility of identifying and evaluating errors in QCA circuitry that goes beyond the simple block majority gate, when identification and optimization are a multivariate problem. The application of our approach to the AND gate will be an interesting future experiment. Additionally, future work will incorporate diagonal QCA instead of only the four lexically contiguous neighbors.

\section{References}

[1]. P. D. Tougaw and C. S. Lent, Logical device implementation using quantum cellular automata, Journal of Applied Physics, 75, $1994,1818-1825$.

[2]. C.S. Lent and P.D. Tougaw, A device architecture for computing with quantum dots, Proc. of the IEEE, 85(4), 1997, 541-557.

[3]. M.T. Niemier, M.J. Kontz, P.M. Kogge, A design of and design tools for a novel quantum dot based microprocessor, Proc. of the $37^{\text {th }}$ Design Automation Conference, Los Angeles, CA, 2000, 227-232.

[4]. W. Porod, Quantum-dot devices and quantum-dot cellular automata," International Journal of Bifurcation and Chaos, 7(10), 1997, 2199-2218.

[5]. G. L. Snider, A. O. Orlov, et. al., Quantum-dot cellular automata, Journal of Vacuum Science Technology A, 17(4), 1999, 13941398.

[6]. A. Fijany and B. N. Toomarian, New design for quantum dots cellular automata to obtain fault tolerant logic gates, Journal of Nanoparticle Research, 3, 2001, 27-37.

[7]. M. B. Tahoori, J. Huang, M. Momenzadeh, F. Lombardi, Testing of quantum cellular automata, IEEE Transactions on Nanotechnology, 3(4), 2004, 432-442.

[8]. C. D. Armstrong, W. M. Humphreys, A. Fijany, The design of fault tolerant quantum dot cellular automata based logic, Proc. of the 11 th NASA Symposium on VLSI Design, 2003.

[9]. E.N. Ganesh, L. Kishore, M. Rangachar, Simulation of quantum cellular automata circuits using neural networks, Proc. of the International Conference on Computational Intelligence and Multimedia Applications, Sivakasi, Tamil Nadu, India, $2007,179-183$.

[10]. L. Kishore, M. Rangachar, Reliability analysis of quantum cellular automata circuits using Bayesian networks, Proc. of the International Conference on Computational Intelligence and Multimedia Applications, Sivakasi, Tamil Nadu, India, 2007, 184-189.

[11]. A. Rangarajan, R. Chellappa, Markov random field models in image processing, in M. Arbib (Ed.), The Handbook of Brain Theory and Neural Networks, (Cambridge, MA: MIT Press, 1995), 564-567.

[12]. S. Bhanja, S. Sakar, Probabilistic modeling of QCA circuits using Bayesian networks, IEEE Transactions on Nanotechnology, 5(6), 2006, 657-670.

[13]. T. Dystart, P. Kogge, Probabilistic analysis of a molecular quantum dot cellular automata adder, Proc. of the $22^{\text {nd }}$ IEEE International Symposium on Defect and Fault-Tolerance in VLSI Systems, Rome, Italy, 2007, 478-486.

[14]. M. Baucells, L. Shapley, Multiperson utility, Games and Economic Behavior, 62, 2008, 329-347. 